\title{
Estrategia corporativa en el ámbito de la sostenibilidad
}

Carlos H. Hurtado Jaramillo ${ }^{1}$, Núria Arimany-Serrat ${ }^{1}$, Xavier Ferràs Hernández ${ }^{1}$, Dulcinea

\author{
Mejide ${ }^{2}$ \\ ${ }^{1}$ Universitat de Vic, ${ }^{2} A G B A R$ (Spain)
}

chhurtado@aqualogy.es, nuria.arimany@uvic.cat, xavier.ferras@uvic.cat, dmeijide@agbar.es

Received July, 2015

Accepted December, 2015

\section{Resumen}

Objeto: El documento pretende fortalecer las estructuras de análisis identificando los aspectos que dentro de una exploración bibliográfica y bajo el criterio de los académicos sean considerados relevantes para la valoración de logros y evolución de la organización en relación a su estrategia de sostenibilidad.

Diseño/metodología/enfoque: Metodología de investigación cualitativa. El estudio parte de una retrospectiva bibliográfica de 25 años, con base en las interpretaciones realizadas por los autores de 90 artículos en el ámbito de la gestión de la sostenibilidad; de esta manera se establece un consenso de opinión respecto a aspectos de marcado interés que debe contener un instrumento de enfoque analítico que permita ajustar la estrategia de sostenibilidad de la organización.

Aportaciones $y$ resultados: Los contextos que alcanzaron un mayor consenso y presentan un marcado interés para los autores son los que asocian aspectos relacionados con los elementos de control necesarios para que exista una adecuada comprobación, inspección, fiscalización o intervención en los procesos de gestión de la sostenibilidad, e integración dentro de la estructura operativa de la empresa como un elemento de cohesión entre los mecanismos de gestión corporativa.

Limitaciones: Los datos cualitativos son una muestra representativa de artículos en un idioma específico, los cuales fueron extractados de dos bases de datos bibliográficos (Scopus e ISI Web Knowledge). Aunque existe un amplio y variado catálogo de artículos 
en diferentes idiomas y en diversas bases de datos, es una exploración exhaustiva que está más allá del alcance de este estudio.

Implicaciones prácticas: El estudio pone de relieve la importancia de asociar los datos recogidos de los diferentes Mecanismos de Gestión Corporativa (MGC) que están directamente involucrados en la estrategia de sostenibilidad, así como los retos que implica el uso de dichos datos para ampliar los beneficios que esta área aporta a la relación de sustentabilidad local-global.

Implicaciones sociales: El estudio propone la implementación del cuadro de mando en las organizaciones, como herramienta de coordinación horizontal para los mecanismos de gestión corporativa; de esta manera se proporciona a los administradores una mirada global de la organización desde diferentes perspectivas; su uso como soporte a la gestión es de alto valor, ya que permite controlar de forma continua si una empresa va a alcanzar los resultados definidos dentro de un plan estratégico de sostenibilidad.

Originalidad / Valor añadido: El estudio ofrece una visión de los aspectos de gestión que de forma iterativa siguen siendo base de estudio por académicos, y que mediante una re-asociación de ideas permite la generación de una propuesta con base en algunos consensos identificados.

Palabras clave: Responsabilidad social corporativa, Sostenibilidad corporativa, Gestión sostenibilidad, Desarrollo sostenible, Estrategia sostenibilidad

Códigos JEL: M14

Title: Corporate strategy in the field of sustainability

\section{Abstract}

Purpose: The paper aims to strengthen the structures of analysis by identifying relevant aspects that within a bibliographic exploration and under academic criteria are considered relevant to assessment of corporate achievements and evolution in relation to its sustainability strategy.

Design/methodology: Qualitative research methodology. The paper is a literature retrospective of 25 years, based on interpretations made by the authors of 90 articles in the field of sustainability management; thus a consensus of opinion is established in 
relation to aspects of strong interest in order to develop an instrument with analytical approach and strategy.

Findings: The contexts that reached greater consensus and have a strong interest for authors are those aspects associated with required elements of "Control" for a proper check, inspection, supervision or intervention in sustainability management processes, and "Integration" within the corporate operational structure as an element of cohesion between corporate management mechanisms.

Research limitations/implications: Qualitative data are a representative sample of articles in a single language, which were extracted from two bibliographic databases (Scopus and ISI Web Knowledge). Although there is a wide and varied catalogue of articles in different languages and in different databases, it is an exhaustive exploration that goes beyond the scope of this study.

Practical implications: The paper highlights the importance of linking data collected from different corporate management mechanism which are directly involved in the sustainability strategy and challenges of using such data to extend the benefits this association brings to the relationship of local-global sustainability.

Social implications: The paper propose the implementation of a scorecard to organizations as horizontal coordination tool for corporate management mechanisms; thus it's provided to managers a global view of the organization from different perspectives; its use as management support has high value, since allowing continuously monitor whether a company will achieve defined outcomes within a strategic sustainability plan.

Originality/value: The paper provides an overview of management aspects that remain iteratively as base of research studies by scholars and that through a reassociation of ideas allows the setting up a proposal regarding at some consensus identified.

Keywords: Corporate social responsibility, Corporate sustainability, Sustainability management, Sustainable development, Sustainability strategy

Jel Codes: M14 


\section{Introducción}

En el proceso de búsqueda para alcanzar la sostenibilidad es necesario que las empresas transformen sus procesos de gestión, donde se consideren cambios en aspectos institucionales, socioculturales, organizacionales y por supuesto tecnológicos, ya que para afrontar problemas persistentes, es necesario abordarlos a través de específicas de redes de trabajo y procesos de toma de decisiones (Loorbach, 2010); así mismo, algunos académicos advierten la demanda por parte de las organizaciones de nuevos mecanismos de análisis para la planeación, orientación y toma de decisiones como elemento fundamental para la construcción de adecuados modelos de crecimiento (Choo, 1996; Bonacchi \& Rinaldi, 2007; Hansen, Grosse-Dunker \& Reichwald, 2009; Loorbach, 2010; Bos-Brouwers, 2010; Ocampo \& Clark, 2014); esto se debe a que el desarrollo global está en una fase donde la innovación está gradualmente enfocándose hacia la generación de herramientas para erigir un mundo más sostenible (Adamczyk, Bullinger \& Möslein, 2012; Gobble, 2014).

Si bien el mundo se halla en una evolución constante en el ámbito de la innovación, existe una problemática entorno al desarrollo de instrumentos con enfoque analítico y de estrategia; en la actualidad no hay consenso frente a la capacidad de los mecanismos de gestión corporativa (MGC) como instrumentos para medir el desempeño de las organizaciones frente a criterios de sostenibilidad (Wiedmann, Lenzen \& Barrett, 2009; Hahn \& Figge, 2011; Montiel \& DelgadoCeballos, 2014); persisten vacíos y debilidades en el desarrollo de instrumentos de gestión, medición y valoración de la sostenibilidad en los negocios donde se demuestre una real integración de aspectos clave de desempeño, comprometiendo así la posibilidad de vincular estrategias sostenibles a las operaciones del día a día (Bonacchi \& Rinaldi, 2007; Lee \& Farzipoor, 2012; Sarvaiya \& Wu, 2014; Galbreath, 2014).

Sin embargo, a pesar de los esfuerzos por parte de las empresas a través de la responsabilidad social corporativa (RSC), la responsabilidad social (RS), desempeño social corporativo (DSC), sostenibilidad corporativa (SC), sistemas integrados de gestión (SIG) y otros mecanismos de gestión corporativa (MGC), aún es difuso el avance que estas han conseguido para demostrar sus logros de forma tangible (Siegel, 2009; Lee, 2011; Lee \& Farzipoor, 2012; Brook \& Pagnanelli, 2014); existe mucho debate en cuanto a la naturaleza exacta del complejo trabajo en describir, medir y demostrar resultados claros en ámbitos de sostenibilidad (Faber, Jorna \& Van Engelen, 2005; Porter \& Kramer, 2006; Ramos \& Caeiro, 2010; Lee \& Farzipoor, 2012; Galbreath, 2014) y que estos sean percibidos como información referencial del mapa general de sostenibilidad que se está construyendo (Bonacchi \& Rinaldi, 2007; León-Soriano, MuñozTorres \& Chalmeta-Rosalen, 2010). 
A este respecto, algunos académicos señalan la importancia de considerar indicadores de desempeño dentro de un único marco de observación o referencia, de tal forma que permita el análisis desde una panorámica holista de la gestión, la identificación de logros, la medición de la evolución y que sirva para ajustar el mapa de ruta en las empresas con relación a su sostenibilidad (Ravetz, 2000; Bonacchi \& Rinaldi, 2007; León-Soriano et al, 2010; Scipioni, Mazzi, Mason \& Manzardo, 2009; Mori \& Christodoulou, 2012; Fegraus et al., 2012; Edgeman \& Williams, 2014).

Como ejemplo de esta problemática se presenta el hecho de que la organización para la Iniciativa de Reporte Global (GRI, siglas en inglés) haya iniciado el proyecto "Reporting 2025: An International Dialogue" con el objetivo de promover una discusión con relación al propósito de los reportes de sostenibilidad y así poder identificarlas principales cuestiones que deberían ser el centro de atención de las empresas para la elaboración de sus agendas de actividades, y así facilitar su gestión y la demostración de resultados.

A pesar de las numerosas publicaciones académicas sobre temas de gestión y medición de la sostenibilidad (Brook \& Pagnanelli, 2014), la realidad es que se ha conseguido un escaso progreso en relación a la planeación y control de la sostenibilidad en general, y en particular en la unificación de instrumentos que realicen una integración real del desempeño en cada una de sus dimensiones (Bonacchi \& Rinaldi, 2007).

Partiendo de esta evidencia, han surgido una serie de preguntas como origen de este estudio:

- ¿Cómo las organizaciones pueden capitalizar los mecanismos de gestión corporativa (MGC) en el diseño de estrategias sostenibles para los procesos de negocio?

- ¿Qué aspectos son limitantes y/o relevantes para que los mecanismos de gestión corporativa (MGC) presenten un enfoque analítico y de estrategia?

- ¿Qué aspectos deben ser resaltados para que desde un panorama integral, se oriente a los gestores a la toma de decisiones entorno al ajuste de la estrategia de sostenibilidad?

El actual desafío que presenta la gestión de la sostenibilidad tiene relación con examinar los elementos confusos que actúan como barrera para la generación de evidencias de desempeño y evolución; en este sentido, algunos académicos insinúan que estos problemas tendrían su origen en posiciones contextuales, en la sensibilización y ambición de las empresas, o en la definición de estrategias dentro de una imagen a largo plazo (Epstein \& Roy, 2001; Marrewijk, 2003; Gaziulusoy, Boyle \& Mcdowall, 2013; Schrettle, Hinz, Scherrer-Rathje \& Friedli, 2014; Brook \& Pagnanelli, 2014). 
Se plantea como objetivo de este documento determinar los aspectos que dentro de una exploración bibliográfica y bajo el criterio de los académicos sean considerados relevantes para la valoración de logros y evolución de la organización en relación a su estrategia de sostenibilidad. Esta decisión se toma considerando que el factor clave en todo proceso innovador es la creatividad, que consiste en sintetizar ideas y nuevos conceptos a partir de la re-estructuración y re-asociación de los ya existentes (Coyne, 1986 citado por Vinayan, Jayashree \& Marthandan, 2012), y a lo señalado por Bisbe (2006), donde establece que las empresas deben basar la innovación en sistemas de coordinación horizontal poco formalizada con el fin de facilitar tanto el análisis como la toma de decisiones.

Por lo tanto, para alcanzar el desafío planteado, es necesario identificar los elementos confusos y solventar las debilidades de gestión corporativa con relación a escenarios de sostenibilidad (Epstein \& Roy, 2001; Dyllick \& Hockerts, 2002; Porter \& Kramer, 2006; Lee \& Farzipoor, 2012; Gaziulusoy et al., 2013; Brook \& Pagnanelli, 2014).

En este sentido, el enfoque de este estudio se encamina a innovar en el fortalecimiento de las estructuras de análisis a partir de la re-organización, asociación o integración de aspectos relevantes dentro de una herramienta de coordinación horizontal; de esta manera se trazará el camino hacia un consenso sobre la idoneidad de los MGC como instrumentos para medir tanto el desempeño de la organización como su evolución dentro de un escenario de sostenibilidad.

Como metodología se planteó la realización de una revisión bibliográfica de 90 artículos publicados entre los años 1989 a 2014 en el ámbito de la gestión y utilizando como base de datos la Web of Knowledge y Scopus; la primera fase se encaminó a determinar los aspectos que han recibido una mayor observación y que se han transformado en bucle de investigación al no lograrse un consenso en relación a cuestiones de valoración tanto de desempeño como de evolución.

Los resultados obtenidos muestran que los aspectos más estudiados durante estos 25 años están asociados a contextos de "Control" (Atributos, Indicadores, Desempeño e Impacto) e "Integración" (Segmentación, Comunicación y Relaciones); en un mediano nivel de interés están "Análisis" (Beneficios, Evolución, Metas y Participación) y "Concepto" (Marco-trabajo, Criterios y Características); la literatura apunta a fallos asociados a la selección, la interpretación y el uso de aspectos esenciales para registrar la evolución y seguimiento a manejos corporativos que puedan ser valorados para determinar su desempeño en sostenibilidad. El valor analítico de este estudio cobra mayor importancia al presentar los aspectos de gestión que de forma iterativa y aislada siguen tratándose en diferentes estudios, y que sin embargo, mediante un razonamiento de forma conjunta permite la generación de propuestas que abordan un mayor número de inquietudes. 
En una segunda fase, se propone una herramienta como alternativa para solventar la falta de comprensión de marcos de trabajo, alcances, propósitos y análisis conjunto que ofrecen los mecanismos de gestión corporativa (MGC) como soporte a la sostenibilidad.

El estudio pone de relieve la importancia de basarse en la asociación de datos recogidos de los diferentes MGC que están directamente involucrados en la estrategia de sostenibilidad, así como los retos que implica el uso de dichos datos para ampliar los beneficios que su análisis aporta a la relación de sustentabilidad local-global.

\section{Marco teórico en relación a la gestión de la sostenibilidad}

A continuación se presenta un marco teórico con base en la retrospectiva bibliográfica realizada, con el fin de dilucidar la percepción existente en relación a los MGC como puntos de referencia de la organización en un escenario de sostenibilidad. La importancia de las prácticas de gestión a nivel empresarial radica en que estas pueden ayudar al consejo de administración a alinear su estrategia corporativa y de negocio desde una perspectiva integradora, cumpliendo al mismo tiempo retos clave en sostenibilidad (Schaltegger \& Burritt, 2010).

\subsection{Desarrollo sostenible, sostenibilidad corporativa y responsabilidad social corporativa}

Durante los últimos treinta años, los conceptos de sostenibilidad corporativa (SC), responsabilidad social corporativa (RSC), desempeño social corporativo (DSC), triple línea base (TBL), sostenibilidad económica (SE), ciudadanía corporativa (CC) y otros mecanismos de sostenibilidad han recibido una atención creciente en la literatura de gestión y práctica corporativa (Shrivastava \& Hart, 1995; Banerjee, 2008; Scherer \& Palazzo, 2011); esto demuestra el interés de las empresas en la inclusión de preocupaciones sociales y medioambientales en el ámbito de sus respectivas operaciones y en sus relaciones con las partes interesadas. Estos mecanismos han surgido desde la perspectiva de establecer un modo de alcanzar un mundo más equilibrado, más humano, más ético y de una manera más transparente para hacer negocios (Marrewijk, 2003; Montiel, 2008; Ross, 2009; Sarvaiya \& Wu, 2014).

Un gran número de académicos están de acuerdo en que el desempeño de la organización está ligado tanto a la sostenibilidad de su crecimiento, como a la de su comportamiento dentro de límites definidos; en este contexto la SC, la RSC, TBL, DSC, SE y CC son términos utilizados 
para referirse al desarrollo sostenible de las organizaciones (Danchev, 2006; León-Soriano et al., 2010; Asif, Searcy, Zutshi, Fisscher, 2013; Bolis, Morioka \& Sznelwar, 2014); en otras palabras, son una práctica de gestión empresarial para asumir las responsabilidades sociales, económicas y ambientales que las organizaciones tienen con sus stakeholders (Montiel, 2008; León-Soriano et al., 2010; Sarvaiya \& Wu, 2014; Bolis et al., 2014).

El debate entre académicos, consultores y ejecutivos está relacionado con desacuerdos entorno a marcos conceptuales y sus alcances en el marco de desarrollo sostenible, dando como resultado diversas definiciones, contextos, enfoques y estrategias (Marrewijk, 2003; Montiel, 2008; Schwartz \& Carroll, 2008; Sarvaiya \& Wu, 2014); la diversidad de definiciones adjuntas a la sostenibilidad en las organizaciones y la falta de especificidad del concepto, ha planteado múltiples controversias y malentendidos en la implementación, gestión y evaluación del desempeño de las prácticas empresariales responsables (Montiel, 2008; Sarvaiya \& Wu, 2014; Bolis et al., 2014).

En la actualidad es posible encontrar todo tipo de términos utilizados para referirse a los informes sociales y medioambientales de las empresas, donde se utilizan títulos tan variados como "Iniciativa de Reporte Global", "Informe Global de Ciudadanía", "Informe de Responsabilidad Corporativa", "Informe de Sostenibilidad Ambiental", "Informe de Sostenibilidad" y "Medio Ambiente y Responsabilidad Social" para referirse a su documento anual, en el cual se resumen sus iniciativas, actividades y frutos alcanzados; con tal variación de informes, es difícil evaluar con exactitud el relativo éxito de la empresa en el logro de la sostenibilidad (Montiel, 2008); en este sentido, se percibe que los contextos confusos suelen generar respuestas inadecuadas, las cuales provocan infructuosos intentos en la búsqueda de generar evidencias de desempeño y avance en sostenibilidad.

Tanto Marrewijk como Montiel, Sarvaiya y Wu están de acuerdo en que estos debates deben ser abandonados ya que sus trabajos demuestran que las conceptualizaciones, su medición y los enfoques convergen hacia el mismo punto (Montiel, 2008); se entiende que estos definen y clarifican la relación entre la empresa y la sociedad, sin negar el éxito que cada una de estos mecanismos ha alcanzado en la gestión corporativa (Sarvaiya \& Wu, 2014); por tanto, la conclusión frente a estos debates radica en su aceptación y ajuste con relación a los niveles de desarrollo, sensibilización y ambición de cada organización (Marrewijk, 2003). Cabe resaltar que aunque existe un cuerpo substancial de literatura orientada a la gestión de la sostenibilidad, indicadores y la medición de su desempeño, no hay un acuerdo consensuado en marcos de trabajo, reglas o estándares para su reconocimiento, sus mediciones y la calificación de los logros en sostenibilidad (Lee \& Farzipoor, 2012; Bolis et al., 2014). 


\subsection{Sostenibilidad y los mecanismos y/o herramientas de gestión corporativa}

Es ampliamente reconocido que tanto los mecanismos de gestión de la sostenibilidad como los sistemas de gestión estandarizados son de carácter voluntario, pero sobresale el hecho que debido a su auge, son hoy día considerados criterios esenciales para los negocios, la imagen y la proyección a futuro (Asif, Searcy, Zutshi \& Ahmad, 2011; Asif et al., 2013).Estos mecanismos están orientados a mejorar el desempeño de la organización mediante el aumento de la satisfacción del consumidor, la reputación, oportunidades de competencia por talento y a la mejora de las estrategias de gestión de riesgos a la seguridad humana y medioambiental, entre otros; de acuerdo con esta perspectiva, es el mercado y sus múltiples actores los que regulan la práctica de la sostenibilidad corporativa (Brammer, Jackson \& Matten, 2012).

La diversidad de las herramientas de gestión corporativa abordan una amplia gama de aspectos, como por ejemplo, la norma de gestión ambiental ISO 14001 y la norma de gestión de la calidad ISO 9001 que han ganado importancia para muchas empresas en las últimas dos décadas (Schaefer, 2007; Banerjee, 2008); la responsabilidad social SA 8000, ISO 26000, SGE-21 y rendición de cuentas 1000 (AA1000: 2000) que son normas sociales y éticas (Asif et al., 2011; Asif et al., 2013); o las OHSAS 18001 que son normas de gestión de la Seguridad y Salud en el Trabajo (Asif et al., 2011; Asif et al., 2013). Es claro que nuevas normas seguirán apareciendo y normas existentes pasara por actualizaciones periódicas, por ejemplo, la norma para la gestión de la energía (SGEn) ISO50001:2011 es un estándar reciente que permite a las organizaciones establecer los sistemas y procesos necesarios para mejorar la eficiencia energética. Si bien estas herramientas se han generado a largo del estos 30 años como soporte de gestión empresarial, de la misma forma estas contribuyen al desempeño de la sostenibilidad mediante la gestión equilibrada de problemas ambientales, sociales y económicos (Scherer \& Palazzo, 2011; Asif et al., 2013; Mohamad, Abdulllah, Mohammad \& Kamaruddin, 2014).

Frandsen, Morsing and Vallentin (2013) señalan que aun con la mayoría de los enfoques de gestión y las herramientas disponibles en la actualidad, aún no existe un enfoque estandarizado o una indiscutible mejor práctica para la implementación de la gestión de la sostenibilidad corporativa; esto se debe principalmente a que el núcleo de la actividad y la situación específica de una empresa requieren de un enfoque a medida en lugar de una solución de carácter universal (Cramer, 2005; Haugh \& Talwar, 2010, citados por Frandsen). Otra característica importante a considerar en este estudio es que el concepto de sostenibilidad es muy polifacético y no bien definido, lo que aumenta la complejidad de su gestión (Siebenhüner \& Arnold, 2007; Scherer \& Palazzo, 2011; Frandsen et al., 2013). 
Además de la amplia gama de interpretaciones teóricas de lo que la gestión de la sostenibilidad implica y lo que debería tratar de lograr, en los últimos 30 años se ha producido una proliferación de mecanismos de gestión que amplían la agenda corporativa; pero estos instrumentos están lejos de plantear una actividad homogénea, debido a cada uno de estos concibe una diversidad de actores, frente a una amplia gama de cuestiones, objetivos y métodos. Este diversidad de instrumentos contemplan además la certificación, la elaboración de informes y el establecimiento de normas; por lo tanto, el panorama se complica aún más debido a que las empresas están cada vez más agobiadas con una progresión de diferentes demandas de información, y al mismo tiempo hacen frente a la falta fiable de referencias sobre los múltiples instrumentos y su potencial para añadir valor a la compleja estructura implementada, con el fin de transmitirlo a sus diferentes grupos de interés (Asif et al., 2013; Mohamad et al., 2014).

\subsection{Benchmarking índices y resultados}

Hoy día es posible identificar una amplia gama de índices a través de los cuales las empresas puedan medir su desempeño en DS; dentro de las agencias de calificación que actualmente tienen alto reconocimiento están Dow Jones Sustainability Index, Carbon Disclosure Project, FTSE4 Good, Global 100, DEFRA (Department of Environment, Food and Rural Affairs), EFFAS (European Federation of Financial Analysts Societies, CRO, Fortune, IFAC (International Federation of Accountants), Sustainability Technologies Initiatives, Green buildings o GRI (Global Reporting Iniciative); estas agencias han elaborado diferentes enfoques y metodologías para calificar la sostenibilidad en las empresas; así mismo, un gran número de indicadores clave han sido determinados por cada una de estas agencias, como base de referencia para valorar el desempeño de los aspectos económicos, ambientales y sociales (Lee \& Farzipoor, 2012; Vigneau, Humphreys \& Moon, 2014; Guo \& Yang, 2014). La publicación de estos índices y la divulgación de sus calificaciones estimulan los mecanismos de reputación y proporcionan una ventaja competitiva a las empresas que están en efecto propiciando activamente los valores sociales y ecológicos (Escrig, Muñoz \& Fernández, 2010; Vigneau et al., 2014; Guo \& Yang, 2014). Desde un punto de vista analítico, las empresas también requieren de estas calificaciones para calibrar y validar sus propios esfuerzos de sostenibilidad; por lo tanto, estas calificaciones deben ser robustas, precisas y creíbles (Wiedmann et al., 2009).

No obstante, si bien existe esta amplia variedad y disponibilidad de agencias de calificación, rankings y premios, las agencias de calificación no cuentan con un consenso sobre cómo desarrollar un mecanismo de medición conjunta; esta falta de acuerdo en relación a indicadores clave de desempeño y metodologías que sean comúnmente aceptables hace más 
compleja la situación y origina una falsa sensación de seguridad en inversores, entidades crediticias y en las partes interesadas (Bonacchi \& Rinaldi, 2007; Escrig et al., 2010; SustainAbility, 2013; Vigneau et al., 2014); esto sucede debido a que el marco de trabajo y calificación de cada una de estas agencias difiere en forma y fondo a la hora de valorar el desempeño de las organizaciones, lo que origina serias dudas sobre el papel y la credibilidad de las calificaciones (Escrig et al., 2010; SustainAbility, 2013; Vigneau et al., 2014; Guo \& Yang, 2014).

Evaluar el desempeño de todas las organizaciones con base en unos criterios estándar es un reto muy complejo, debido principalmente a las diferentes perspectivas de sostenibilidad de los stakeholders, las agencias de calificación y las empresas; esto principalmente se debe a la significativa heterogeneidad de la organizaciones en tamaño, actividad, área de influencia e intereses particulares (Riccaboni \& Leone, 2011; Scherer \& Palazzo, 2011; Edgeman \& Williams, 2014; Vigneau et al., 2014); estas afirmaciones complementan ampliamente la premisa de la teoría del desarrollo internacional que señala la existencia de aspectos únicos y condiciones específicas de las economías en desarrollo, las cuales exigen soluciones diferentes a las que podrían ser implementadas en las economías desarrolladas.

El proyecto "Rate The Raters" realizado por SustainAbility (organización experta desde 1987 en innovación y ofrecer soluciones para hacer los negocios y mercados sostenibles) entre los años 2010 y 2013, tuvo como objetivo comprender mejor el universo de las calificaciones de sostenibilidad con el fin de mejorar la calidad y transparencia de estas calificaciones; en el informe final presentado en el 2013, se reconocen más de 100 índices de los cuales solo 21 existían antes del año 2000, lo que demuestra la enorme proliferación de clasificaciones en los últimos años (SustainAbility, 2013) debido a la necesidad de las empresas en ganar reputación y ventaja competitiva a través de sus valores sociales y ecológicos (Escrig et al., 2010; Vigneau et al., 2014; Guo \& Yang, 2014). El proyecto proporciono perspectivas que pueden ayudar a las empresas, los inversores y las partes interesadas a dar más sentido a las metodologías y valor a las calificaciones. Pero aún existen muchas cuestiones en relación a los aspectos de valoración, los procesos de evaluación y entorno a los marcos de trabajo que se deben emplear al momento de calificar el desempeño de las empresas; Lee \& Farzipoor (2012) señalan la importancia de desarrollar marcos de trabajo con claros principios, criterios, metas y alcances para poder medir la sostenibilidad a nivel corporativo en términos operacionales y que estos a su vez sirvan para el análisis de la evolución de los datos. 


\subsection{Informes corporativos de sostenibilidad}

A pesar de los continuos llamamientos para la adopción de prácticas de gestión de la sostenibilidad a nivel corporativo, su incorporación a nivel operativo en las empresas sigue siendo a menudo difuso, poco preciso y más superficial que efectivo (Figge, Hahn, Schaltegger \& Wagner, 2002; Siegel, 2009; Lee, 2011; Lee \& Farzipoor, 2012; Mohamad et al., 2014); las razones que algunos académicos atribuyen a esta falta de integración son principalmente dos; la primera, que las empresas no entienden cómo abordar, medir y gestionar los aspectos de sostenibilidad de una manera sistemática (Briassoulis, 2001; Ramos \& Caeiro, 2010; Hahn \& Figge, 2011; Aguinis \& Glavas, 2012); la segunda, hace relación a la insuficiencia de herramientas de análisis disponibles para apoyar las prácticas de gestión de la sostenibilidad en términos concretos de operación y estrategia (Choo, 1996; Briassoulis, 2001; Bonacchi \& Rinaldi, 2007; Hansen et al., 2009; Loorbach, 2010; Bos-Brouwers, 2010; Ocampo \& Clark, 2014; Bolis et al., 2014).

Así mismo, a pesar de las numerosas publicaciones académicas sobre temas de gestión y medición de la sostenibilidad (Epstein \& Roy, 2001; Figge et al, 2002; Faber et al., 2005; Bonacchi \& Rinaldi, 2007; León-Soriano et al., 2010; Asif et al., 2011; Asif et al., 2013; Brook \& Pagnanelli, 2014) y aunque los reportes de sostenibilidad se hayan convertido en una cuestión de alta importancia, la realidad es que se ha conseguido un escaso progreso en relación a la planeación y control de la sostenibilidad en general, y en particular en la unificación de instrumentos que realicen una integración real del desempeño en cada una de sus dimensiones (Bonacchi \& Rinaldi, 2007).

En las últimas décadas se ha avanzado mucho en la elaboración de normas y directrices internacionales para la certificación de la gestión y mejora continua de aspectos de calidad, medio ambiente, eficiencia energética, seguridad y salud en el trabajo de igual forma se ha avanzado en aspectos de certificación de aspectos éticos y de responsabilidad social (Schaefer, 2007; Banerjee, 2008; Asif et al., 2011; Asif et al., 2013); a pesar de estos esfuerzos, el principal obstáculo de estas normas y directrices a la hora de ayudar a las empresas a implementar los enfoques de sostenibilidad a nivel corporativo radica en que siguen siendo sugerencias y recomendaciones generales sobre cómo las empresas deben tomar en cuenta los objetivos de sostenibilidad de la organización en la planeación de sus actividades (Asif et al., 2013); por este motivo, los informes corporativos de sostenibilidad son vistos como difusos, poco precisos y poco creíbles, donde la evidencia de la práctica parece mostrar una realidad diferente a la que se percibe (Kolk, 2004; Moneva, Archel \& Correa, 2006).

En este sentido, aun con la amplia variedad de metodologías empleadas por las agencias de calificación, con la extensa gama de indicadores clave y con la diversidad de herramientas de 
gestión corporativa, es difícil responder o establecer claramente en los informes corporativos el grado de cumplimiento de una empresa en sostenibilidad; la identificación y medición de la gestión de sostenibilidad corporativa sigue siendo muy difícil debido a los altos niveles de incertidumbre metodológica y que a menudo es difícil cuantificar los impactos generados (Briassoulis, 2001; Lee \& Farzipoor, 2012; Montiel \& Delgado, 2014).

\section{Materiales y Métodos}

\subsection{Diseño de la investigación}

Debido al limitado número de referencias disponibles en la literatura sobre la identificación de los aspectos de atención recurrentes en el campo de la gestión de la sostenibilidad; para este estudio se concibió un diseño de investigación cualitativo de naturaleza exploratoria; el proceso de análisis residió en la selección y clasificación de la información, generando diferentes composiciones de categorías según temas o tipologías a analizar; finalmente esta composición debe facilitar la interpretación de los resultados mediante la comparación, cruce o confrontación de los datos (Creswell, 2007). Por lo tanto, para la construcción de la tabulación se realizó una revisión bibliográfica con enfoque en dos preguntas específicas "Que aspectos son recurrentes como objeto de investigación" y "Cuales son los aspectos de mayor señalamiento por parte de los académicos".

Se parte de la elaboración de una lista a partir de aspectos de interés que emergen de los artículos, determinando las tendencias que se han presentado a lo largo del periodo de estudio. En un primer paso, se elaboró una selección global de artículos relacionados con términos que aplicaban exclusivamente con la gestión de la sostenibilidad; en un segundo paso, se realizó una clasificación de los artículos de acuerdo a las terminologías más reconocidas y utilizadas; por último, se extrajeron los aspectos que convergen dentro de cada categoría y su porcentaje de recurrencia.

\subsection{Estableciendo la literatura base}

Con el fin de estructurar el conocimiento actual que vincula los temas de gestión de la sostenibilidad con materias relacionadas a mecanismos, herramientas, desempeño, debilidades y vacíos, se realizó una revisión bibliográfica de artículos académicos empleando la base de datos tanto del Scopus como del ISI Web Knowledge en el mes de junio de 2014.Un conjunto de artículos fue creado por medio de un proceso que englobó los siguientes pasos: 
- Elección de palabras clave relacionadas con gestión, sostenibilidad, desempeño, herramientas, mecanismos.

- Selección de artículos desde la base de datos de Scopus por medio de una búsqueda estructurada usando las palabras clave predefinidas; únicamente fueron extraídos los artículos de revistas en ingles en un periodo que comprende de 1989 a 2014.

- En los campos de búsqueda serelacionó términos que aplicaban exclusivamente con la gestión de la sostenibilidad (DS, RSC, RS, DSC, SC, TBL, CC, SE).

- Se revisó principalmente el título, palabras clave y los resúmenes de los artículos identificados.

- Los artículos se clasificaron previamente de acuerdo con su relevancia a este estudio, y se agruparon en tres grandes bloques según su orientación (DS, RSC, SC).

- Como resultado del refinamiento, se parte de una literatura base de 90 artículos repartida en 3 subconjuntos de 30 artículos cada uno.

\subsection{Clasificación de los artículos de acuerdo a la literatura}

El desarrollo sostenible (DS) es un concepto bastante amplio que tiene implicaciones importantes en el ámbito corporativo, ya que las organizaciones deben enfrentarse no solo a retos relacionados con presiones de desempeño financiero sino también a las crecientes expectativas de la sociedad para que actúen de forma más responsable en complejos contextos sociales, económicos y ambientales; por tanto, al abordar temas de DS en el entorno corporativo, se identificó dos grandes terminologías donde los conceptos más reconocidos y utilizados en los artículos explorados fueron los de RSC y SC.

El desarrollo sostenible (DS) es un concepto bastante amplio que tiene implicaciones importantes en el ámbito corporativo ya que los esfuerzos no solo deben estar destinados a promover su crecimiento económico, dado que están bajo un severo escrutinio por el impacto que generan en la sociedad y en los ecosistemas actuales, así como también por su repercusión en las generaciones futuras (Bonacchi \& Rinaldi, 2007); en la actualidad, las organizaciones deben abordar multifacéticos y complejos contextos sociales, económicos y ambientales, enfrentándose a retos relacionados con presiones de desempeño financiero y a las crecientes expectativas de la sociedad para que actúen de forma más responsable; por tanto, al abordar temas de DS en el entorno corporativo, se identificó dos grandes terminologías donde los conceptos más reconocidos y utilizados en los artículos explorados fueron los de RSC y SC. 
Aunque la RSC, SC y SD son considerados por algunos académicos como conceptos diferentes, hay una clara coincidencia, interrelación e interconexión entre estos conceptos (Marrewijk, 2003; Montiel, 2008; Sarvaiya \& Wu, 2014); bajo esta consideración, parece ventajoso a nivel práctico explorarlos simultáneamente, por consiguiente, se planteó como metodología para este estudio un análisis de los términos de forma individual y luego de manera conjunta. Desde el conjunto de artículos seleccionados, cada documento fue leído en su totalidad y se clasifico por medio de los siguientes parámetros:

- Tipo de concepto con el que se relaciona (RSC, DS, SC)

- Fecha de publicación del estudio

- Principal(es) aspecto(s) de estudio

- Herramientas y/o mecanismos descritos

Por lo tanto, la base de la literatura final está compuesta por 90 artículos repartidos en 3 subgrupos (Ver Tabla 1). Este documento ofrece un panorama global de los aspectos que han sido estudiados reiteradamente en el periodo comprendido entre 1989 y 2014, y que al ser tópicos de estudio de forma recurrente, son considerados relevantes para demostrar el desempeño que realizan las organizaciones en la gestión de su sostenibilidad.

\begin{tabular}{|l|c|c|}
\hline \multicolumn{1}{|c|}{ Subgrupos } & No Artículos & Años \\
\hline Desarrollo Sostenible & 30 & $1989-2014$ \\
\hline Responsabilidad Social Corporativa & 30 & $2000-2014$ \\
\hline Sostenibilidad Corporativa & 30 & $2000-2014$ \\
\hline
\end{tabular}

Tabla 1. Clasificación de los subgrupos con base en su reconocimiento

\section{Resultados y discusión}

\subsection{Resultados}

El producto del proceso de revisión bibliográfica se halla en la Tabla 2; en ésta se presenta el listado de aspectos identificados en la exploración de los 90 artículos y que han sido considerados como relevantes o condicionantes por los mismos autores en el desarrollo de sus propios estudios; con base en esta información, se han definido estos aspectos como punto de partida para su posterior análisis.

Se agrupó un total de 29 aspectos que han sido señalados y/o relacionados como relevantes frente al tema principal de estudio en cada uno de los documentos explorados; de igual forma se tuvo en consideración los aspectos que por similitud se hallaban dentro de la misma franja 
de relación o de equivalencia (sinónimos); con el fin de determinar características comunes dentro del conjunto total de aspectos identificados, se decidió concentrarlos por carácter, contexto u orientación; finalmente de este proceso surgieron 6 categorías: Conceptual, Referencia, Gerencia, Control, Análisis e Integración. (Ver en Tabla 2).

\begin{tabular}{|c|c|c|c|c|}
\hline CARÁCTER & ASPECTO & No & SUBGRUPOS & CLAVE \\
\hline \multirow[t]{4}{*}{ Conceptual } & Ambigüedad conceptual & 1 & DS, RSC, SC & Ambigüedad \\
\hline & Características indefinidas & 2 & DS, RSC, SC & Características \\
\hline & Desconocimiento del marco de trabajo & 3 & DS, RSC, SC & Marco-trabajo \\
\hline & Criterios a considerar & 4 & $\mathrm{DS}, \mathrm{RSC}, \mathrm{SC}$ & Criterios \\
\hline \multirow[t]{3}{*}{ Referencia } & Criterios de calificación & 5 & $\mathrm{DS}, \mathrm{RSC}, \mathrm{SC}$ & Calificación \\
\hline & Condiciones de frontera - parámetros limite & 6 & DS, RSC, SC & Parámetros \\
\hline & Credibilidad de los índices & 7 & DS, RSC, SC & Índices \\
\hline \multirow[t]{8}{*}{ Gerencia } & Objetivos concisos & 8 & DS, RSC, SC & Objetivos \\
\hline & Excesiva flexibilidad & 9 & $\mathrm{DS}, \mathrm{RSC}, \mathrm{SC}$ & Flexibilidad \\
\hline & Intereses difusos & 10 & $\mathrm{DS}, \mathrm{RSC}, \mathrm{SC}$ & Intereses \\
\hline & Compromisos concretos & 11 & DS, RSC, SC & Compromisos \\
\hline & Gobernanza débil & 12 & $\mathrm{DS}, \mathrm{RSC}, \mathrm{SC}$ & Gobernanza \\
\hline & Falta de estrategia & 13 & DS, RSC, SC & Estrategia \\
\hline & Elementos de accionamiento o conductores & 14 & $\mathrm{DS}, \mathrm{RSC}, \mathrm{SC}$ & Conductores \\
\hline & Hojas de ruta - planeación & 15 & $\mathrm{DS}, \mathrm{RSC}, \mathrm{SC}$ & Planeación \\
\hline \multirow[t]{5}{*}{ Control } & Propiedades o atributos de análisis & 16 & DS, RSC, SC & Atributos \\
\hline & Disponibilidad de indicadores & 17 & $\mathrm{DS}, \mathrm{RSC}, \mathrm{SC}$ & Indicadores \\
\hline & Ámbitos de actividades & 18 & DS, RSC, SC & Ámbitos \\
\hline & Impacto generado & 19 & $\mathrm{DS}, \mathrm{RSC}, \mathrm{SC}$ & Impacto \\
\hline & Unidades de referencia de desempeño & 20 & DS, RSC, SC & Desempeño \\
\hline \multirow[t]{5}{*}{ Análisis } & Beneficios poco claros & 21 & $\mathrm{DS}, \mathrm{RSC}, \mathrm{SC}$ & Beneficios \\
\hline & Condiciones de evolución & 22 & $\mathrm{DS}, \mathrm{RSC}, \mathrm{SC}$ & Evolución \\
\hline & Análisis de escenarios & 23 & DS, RSC, SC & Escenarios \\
\hline & Hitos-metas alcanzados & 24 & $\mathrm{DS}, \mathrm{RSC}, \mathrm{SC}$ & Metas \\
\hline & Participación & 25 & DS, RSC, SC & Participación \\
\hline \multirow{4}{*}{ Integración } & Segmentación grupos de interés & 26 & DS, RSC, SC & Segmentación \\
\hline & Integración en la estructura & 27 & $\mathrm{DS}, \mathrm{RSC}, \mathrm{SC}$ & Integración \\
\hline & Comunicación/dialogo & 28 & DS, RSC, SC & Comunicación \\
\hline & Relaciones de vinculo & 29 & $\mathrm{DS}, \mathrm{RSC}, \mathrm{SC}$ & Relaciones \\
\hline
\end{tabular}

Tabla 2. Aspectos considerados relevantes o condicionantes dentro de la exploración bibliográfica

Los 29 aspectos están relacionados en mayor o menor grado dentro de los 3 subgrupos creados a partir de la clasificación de conceptos con mayor reconocimiento en la literatura; para facilitar la visualización gráfica del consenso de los tres subgrupos en relación a cada aspecto, se ha designado a cada uno su respectiva palabra clave.

El levantamiento de la información se realizó mediante una tabulación de tipo binario, en la cual se registraba con un "1" los aspectos que habían sido relacionados en un mismo artículo y con un "0" los que no. Mediante este proceso se pudo identificar el número de veces que se relacionaba un aspecto dentro de los diferentes artículos explorados. 
Para la tabulación de los datos se utilizó un cuadro de análisis individual por cada aspecto y su cuantificación se realizó con el estadístico de porcentaje cuya fórmula es:

$$
\%=\frac{F * 100}{N}
$$

$\%=$ Porcentaje de relación de un aspecto, dentro del total del subgrupo

$F \quad=$ Número de veces que se repite el aspecto dentro del subgrupo

$100=$ Constante

$N=$ Número total de artículos en cada subgrupo

El proceso se repitió en cada uno de los subgrupos creados, de esta forma se pudo constatar cuales aspectos representan un "marcado interés" dentro de cada subgrupo; posteriormente, se realizó una representación gráfica en la que se compilo los resultados obtenidos dentro de los tres subgrupos (Figura 1 ).

En la Figura 1 se presenta el porcentaje de relación de cada aspecto en tres diferentes colores, según cada subgrupo; así mismo se demarcan las 6 categorías en las que se concentraron por carácter, contexto u orientación. Para el análisis de la gráfica se estimaron dos niveles de importancia, el de primer nivel para los aspectos que tenían un porcentaje igual o superior al $60 \%$ y el segundo nivel para los aspectos con un porcentaje igual o superior al $45 \%$.

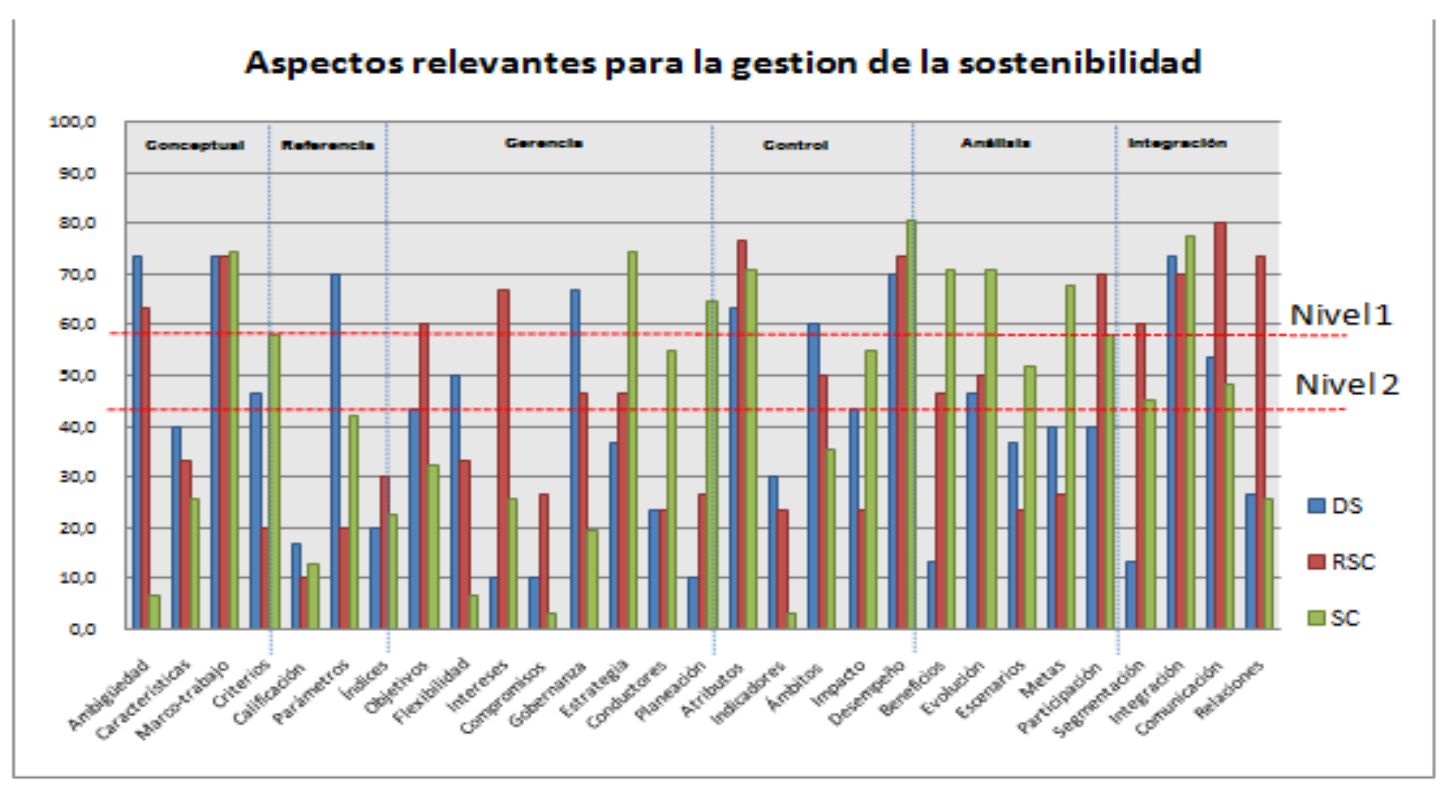

Figura 1. Tabulación de aspectos relevantes para la gestión de la sostenibilidad en cada subgrupo 


\subsection{Aspectos relevantes para la gestión de acuerdo a los subgrupos}

Para obtener una mayor visualización del panorama de consenso alcanzado entre los autores de cada subgrupo, en concordancia a los aspectos que cada uno ha considerado relevante dentro de su estudio, se presentan en primer lugar los aspectos que han sido considerados relevantes dentro de los tres subgrupos tanto en el primer como en el segundo nivel; a continuación los aspectos relevantes para dos subgrupos y por último los relevantes dentro de un solo subgrupo.

\subsubsection{Aspectos relevantes para los tres subgrupos}

Como punto importante a resaltar en la Figura 1 se observa el conjunto de aspectos que dentro de los tres subgrupos presentan un alto porcentaje de consenso frente a la muestra total; se aprecia como estos traspasan el primer nivel de importancia con valores mayores al $60 \%$. Como se advierte, estos aspectos conciernen a contextos de "Concepto", "Control" e "Integración" y hacen alusión al desconocimiento del marco de trabajo a seguir por parte de las empresas ("Marco-trabajo"), las propiedades o atributos de análisis que deben controlarse ("Atributos"), las unidades de referencia en las cuales se mide el desempeño alcanzado ("Desempeño") y finalmente las dificultades de integración a la estructura operativa de la organización ("Integración").

Superando el Nivel 2 se hallan los aspectos de "Evolución" y "Comunicación"; el primero ofrece a la organización un análisis del avance alcanzado con la gestión realizada dentro de una estrategia trazada (Schaltegger, Beckmann \& Hansen, 2013; Montiel \& Delgado, 2014), y el segundo permite integrar la gestión de la sostenibilidad a la estructura de la empresa mediante el dialogo con sus stakeholders (Sarvaiya \& Wu, 2014; Galbreath, 2014).

\subsubsection{Aspectos relevantes para dos subgrupos}

Es posible ver en la Figura 1 aspectos que hacen parte de dos subgrupos y superan el $60 \%$ de relevancia; dentro de estos se señala el aspecto "Ambigüedad" con una marcada importancia tanto en DS como en RSC; esto se debe principalmente a que estos términos tienen un mayor reconocimiento a nivel global, y por tanto, han sido más criticados por sus inconsistencias, así como por la falta de resultados claros y respuestas satisfactorias (Alonso \& Azofra, 2008).

Superando el Nivel 2 se observan aspectos dentro delos 6 contextos señalados, como lo son:Conceptual ("Criterios"), Gerencia ("Gobernanza", "Estrategia"), Control ("Ámbitos"), 
Análisis ("Beneficios", "Participación") e Integración ("Segmentación"). Una de las características que resulta interesante dentro de estos conjuntos, corresponde con algunos aspectos que llegan a superar el primer nivel de relevancia; dentro de estos resaltan: "Gobernanza" y "Ámbitos" (DS), "Estrategia" y "Beneficios" (SC) y "Participación" (RSC). Puede por tanto deducirse que lo autores consideran de forma particular, que estos aspectos presentan un marcado interés en función de cada subgrupo.

\subsubsection{Aspectos relevantes dentro de un subgrupo}

En la Figura 1 se pueden ver aspectos que dentro de un subgrupo superan el primer nivel de relevancia $(60 \%)$ y aunque no exista un tipo de consenso entre los autores que representan los subgrupos, es necesario considerar que presentan un significativo grado de importancia para requisitos específicos dentro de cada MGC. Los aspectos que superan el primer nivel dentro de cada subgrupo y contexto son: para DS "Parámetros"; en RSC "Intereses" y "Relaciones"; en SC "Planeación" y "Metas". En un segundo nivel se encuentran: para RSC "Objetivos"; para SC "Conductores", "Impacto" y "Escenarios".

\subsection{Contextos con mayor relevancia para la gestión}

Para facilitar el análisis de los aspectos se equipararon características comunes dentro del conjunto total de aspectos identificados y se agruparon según su carácter, contexto u orientación; de este proceso surgieron 6 categorías. (Ver la Tabla 2)

Los contextos que alcanzaron un mayor consenso y presentan un marcado interés para los autores son los que asocian aspectos relacionados con los elementos de "Control" necesarios para que exista una adecuada comprobación, inspección, fiscalización o intervención en los procesos de gestión de la sostenibilidad, e "Integración" dentro de la estructura operativa de la empresa como un elemento de cohesión entre los MGC.

Los contextos "Conceptuales" y "Análisis" están en un segundo grado de interés; estos reúnen aspectos que dan mayor claridad a las características, marcos y criterios que deben ser analizados dentro de la gestión y que determinan la evolución que se ha alcanzado en el escenario de sostenibilidad corporativa. Por último están los contextos "Gerencia" y "Referencia", los cuales tienen una relación directa con la toma de decisiones de la dirección para la elaboración de las estrategias con relación a parámetros de referencia que se deseen alcanzar. 


\subsection{Discusión}

En las organizaciones existe múltiples MGC orientados hacia la gestión de la sustentabilidad, donde algunos poseen reconocimiento mundial gracias a ser normas y directrices (ISO's, EMAS, SGE-21, etc) que garantizan la buena ejecución de funciones en toda la estructura operativa de la empresa, mediante procedimientos que aseguran que características positivas de gestión sean controladas y evaluadas dentro de un mismo nivel de exigencia (Asif et al., 2013; Mohamad et al., 2014); por tanto, contextos como "Control" están en gran parte cubiertos gracias a normativas de gestión altamente definidas.

Si bien se cuenta con múltiples MGC, estas se limitan de forma individual a establecer un conjunto de indicadores que varían en función de las corrientes que los tratan, ya sea entornos de calidad (ISO 9001), de seguridad y salud en el Trabajo (OHSAS 18001), de responsabilidad social y ética (SA 8000, ISO 26000, SGE-21, RSC) o energéticas (ISO50001); de hecho, algunas organizaciones se enfocan en los reportes de sustentabilidad tomando como base los MGC de forma aislada y poco cohesiva ("Integración"); por lo tanto es posible deducir que la patología recurrente al interior de las empresas tiene relación con un fallo en el tratamiento de todos los MGC que son soporte para la gestión integrada y equilibrada de la sustentabilidad.

El contexto "Conceptual" resalta los vacíos asociados a la falta de comprensión de las condiciones, alcance, propósito y esquemas de trabajo en los mecanismos de gestión de la sostenibilidad en las empresas (DS, RSC, SC); en este sentido, se confirma lo señalado por algunos académicos cuando afirman que la falta de claridad y sentido en los conceptos dificultan la labor de análisis ("Análisis") del desempeño de la organización (Isaksson, 2006; Hansen et al., 2009; Bos-Brouwers, 2010; Asif et al., 2011; Ocampo \& Clark, 2014). Es factible considerar que tanto la parte "Conceptual" como sucede con "Control" podrían quedar englobados (en cierta medida) por las características, marcos y criterios de los MGC, de esta forma se daría mayor sentido y claridad al ámbito de la gestión de sostenibilidad y a su vez facilitar la labor de análisis.

\subsection{Propuesta}

La gestión de la sostenibilidad en las prácticas empresariales no sólo requiere de la integración y control de los MGC o de un simple examen de los efectos de sus iniciativas y sus resultados (es decir, desempeño), también requiere que las directivas puedan obtener una buena comprensión de los impactos de sus procesos en los entornos externo e interno, de su avance dentro de un mapa referencial y además tengan como soporte para la toma de decisiones una 
herramienta de análisis donde se visualice un panorama general de gestión (Schaltegger et al., 2013; Montiel \& Delgado, 2014; Katrin \& Kehrbach, 2014).

Aunque es reconocido por algunos académicos la reiterada dificultad de establecer criterios y unidades de referencia para medir el desempeño de sostenibilidad en el contexto de las decisiones corporativas (Briassoulis, 2001; Hjortha \& Bagheria, 2006), es necesario recordar quela innovación dinámica es el resultado de sintetizar ideas y nuevos conceptos a partir de la re-estructuración y re-asociación de los ya existentes (Coyne, 1986 citado por Vinayan et al., 2012). Partiendo de esta premisa, se realizó un análisis de herramientas que pudieran servir como mapas referenciales para la empresa en la búsqueda de sus objetivos de sostenibilidad; como resultado, este estudio propone una alternativa para solventar los aspectos señalados en la Tabla 2.

\begin{tabular}{|c|c|c|c|c|c|c|c|c|c|}
\hline \multicolumn{4}{|c|}{ CUADRO DE MANDO } & $\begin{array}{l}\text { RSC/ } \\
\text { SC }\end{array}$ & $\begin{array}{c}\text { ISO } \\
14001\end{array}$ & $\begin{array}{c}\text { ISO } \\
20121\end{array}$ & EMAS & $\begin{array}{c}\text { ISO } \\
9001\end{array}$ & $\begin{array}{c}\text { ISO } \\
26000\end{array}$ \\
\hline \multicolumn{2}{|c|}{ REPORTES } & \multicolumn{2}{|c|}{ DIMENSION } & KPI & KPI & KPI & KPI & KPI & KPI \\
\hline \multirow{12}{*}{$\begin{array}{l}\text { DOW JONES } \\
\text { SUSTAINABILITY }\end{array}$} & \multirow{12}{*}{$\begin{array}{c}\text { GLOBAL } \\
\text { REPORTING } \\
\text { INICIATIVE }\end{array}$} & \multirow{4}{*}{ Social } & KPI & $\mathrm{X}$ & $\mathrm{x}$ & $\mathrm{X}$ & $\mathrm{X}$ & $\mathrm{X}$ & $X$ \\
\hline & & & KPI & $x$ & $X$ & $\mathrm{X}$ & $x$ & $\mathrm{X}$ & $\mathrm{X}$ \\
\hline & & & KPI & $x$ & $\mathrm{X}$ & $\mathrm{X}$ & $\mathrm{X}$ & $\mathrm{X}$ & $\mathrm{X}$ \\
\hline & & & KPI & $\mathrm{X}$ & $\mathrm{X}$ & $\mathrm{X}$ & $\mathrm{X}$ & $\mathrm{X}$ & $\mathrm{X}$ \\
\hline & & \multirow{4}{*}{ Económica } & KPI & $x$ & $\mathrm{X}$ & $x$ & $\mathrm{X}$ & $\mathrm{X}$ & $\mathrm{X}$ \\
\hline & & & KPI & $\mathrm{X}$ & $\mathrm{X}$ & $\mathrm{X}$ & $\mathrm{X}$ & $\mathrm{X}$ & $\mathrm{X}$ \\
\hline & & & KPI & $\mathrm{X}$ & $\mathrm{X}$ & $\mathrm{X}$ & $\mathrm{X}$ & $\mathrm{X}$ & $\mathrm{X}$ \\
\hline & & & KPI & $x$ & $x$ & $\mathrm{X}$ & $\mathrm{X}$ & $\mathrm{X}$ & $x$ \\
\hline & & \multirow{4}{*}{ Ambiental } & KPI & $\mathrm{X}$ & $\mathrm{X}$ & $\mathrm{X}$ & $\mathrm{X}$ & & \\
\hline & & & KPI & $\mathrm{X}$ & $\mathrm{X}$ & $x$ & $\mathrm{X}$ & & \\
\hline & & & KPI & $\mathrm{X}$ & $\mathrm{X}$ & $\mathrm{X}$ & $\mathrm{X}$ & & \\
\hline & & & KPI & $\mathrm{X}$ & $\mathrm{X}$ & $\mathrm{X}$ & $\mathrm{X}$ & & \\
\hline \multicolumn{4}{|c|}{ CUADRO DE MANDO } & $\begin{array}{c}\text { SGE- } \\
21\end{array}$ & $\begin{array}{c}\text { ISO } \\
50001\end{array}$ & $\begin{array}{l}\text { OHSAS } \\
18001\end{array}$ & SA8000 & \begin{tabular}{|c} 
ISO \\
55000
\end{tabular} & NIIF \\
\hline \multicolumn{2}{|c|}{ REPORTES } & \multicolumn{2}{|c|}{ DIMENSION } & KPI & KPI & KPI & KPI & KPI & KPI \\
\hline \multirow{12}{*}{$\begin{array}{l}\text { DOW JONES } \\
\text { SUSTAINABILITY }\end{array}$} & \multirow{12}{*}{$\begin{array}{l}\text { GLOBAL } \\
\text { REPORTING } \\
\text { INICIATIVE }\end{array}$} & \multirow{4}{*}{ Social } & KPI & $\mathrm{X}$ & & $\mathrm{X}$ & $\mathrm{X}$ & & \\
\hline & & & KPI & $\mathrm{X}$ & & $\mathrm{X}$ & $\mathrm{X}$ & & \\
\hline & & & KPI & $\mathrm{X}$ & & $\mathrm{X}$ & $\mathrm{X}$ & & \\
\hline & & & KPI & $x$ & & $\mathrm{X}$ & $\mathrm{X}$ & & \\
\hline & & \multirow{4}{*}{ Económica } & KPI & $\mathrm{X}$ & $\mathrm{X}$ & & & $\mathrm{X}$ & $\mathrm{X}$ \\
\hline & & & KPI & $\mathrm{X}$ & $\mathrm{X}$ & & & $\mathrm{X}$ & $\mathrm{X}$ \\
\hline & & & KPI & $\mathrm{X}$ & $\mathrm{X}$ & & & $\mathrm{X}$ & $\mathrm{X}$ \\
\hline & & & KPI & $\mathrm{X}$ & $\mathrm{X}$ & & & $\mathrm{X}$ & $\mathrm{X}$ \\
\hline & & \multirow{4}{*}{ Ambiental } & KPI & & $\mathrm{X}$ & $\mathrm{X}$ & & & \\
\hline & & & KPI & & $\mathrm{X}$ & $\mathrm{X}$ & & & \\
\hline & & & KPI & & $\mathrm{X}$ & $\mathrm{X}$ & & & \\
\hline & & & KPI & & $\mathrm{X}$ & $\mathrm{X}$ & & & \\
\hline
\end{tabular}

KPI = Indicadores clave de rendimiento $\mathrm{AA} 1000=\mathrm{RSC}$

Tabla 3. Cuadro de mando como herramienta de cohesión de los MGC 
Se parte de la recomendación efectuada por Bisbe (2006) donde señala que las organizaciones deben basar la integración en sistemas de coordinación horizontal poco formalizada, debido a que "el uso interactivo de sistemas de control comporta discusiones cara a cara a incertidumbres estratégicas... y aumenta significativamente el grado en que estas iniciativas se traducen efectivamente en resultados". Por tanto, se propone la asociación en red de todos los MGC como una estructura de soporte global, esta red integrada actuaría como una herramienta de coordinación horizontal (Cuadro de Mando) para la gestión de la sostenibilidad, aprovechando la organización existente de los sistemas implantados y evitando la necesidad de crear uno nuevo. De esta manera se traza el camino hacia un consenso sobre la idoneidad de los MGC como instrumentos para medir tanto el desempeño de la organización como su evolución dentro de un escenario de sostenibilidad. Es poco probable que se produzcan mayores progresos en sostenibilidad, hasta que no se diseñen maneras eficaces para medir el desempeño de las acciones corporativas emprendidas en este tema (Atlee \& Kirchain, 2006). El cuadro de mando es una herramienta que está orientada a la cohesión de todas los MGC como forma de equilibrar los elementos de sustentabilidad y a su vez sirve como soporte analítico al ofrecer una perspectiva completa del desempeño de las acciones corporativas emprendidas por la organización (Ver Tabla 3).

\begin{tabular}{|c|c|}
\hline CARÁCTER & VENTAJAS \\
\hline Conceptual & $\begin{array}{l}\text { Con los MGC se da mayor claridad a las características, marcos y criterios que deben } \\
\text { ser analizados. }\end{array}$ \\
\hline Referencia & $\begin{array}{l}\text { La integración de los criterios de evaluación de los MGC reduce las lagunas y mejora } \\
\text { la coherencia y credibilidad. }\end{array}$ \\
\hline Gerencia & $\begin{array}{l}\text { Partiendo de la implementación de una estrategia única para el logro de objetivos de } \\
\text { manera conjunta. }\end{array}$ \\
\hline \multirow{3}{*}{ Control } & $\begin{array}{l}\text { Asociar los indicadores o combinaciones de indicadores de los MGC proporciona una } \\
\text { señal más clara de progreso. }\end{array}$ \\
\hline & $\begin{array}{l}\text { Estandariza en gran medida ámbitos de actividades y propiedades de análisis. Esto } \\
\text { permite la comparación de valores, indicadores de objetivos, valores de referencia, } \\
\text { intervalos, umbrales o dirección de las tendencias. }\end{array}$ \\
\hline & $\begin{array}{l}\text { Se mejoran las métricas de rendimiento y la disponibilidad de información para } \\
\text { rastrear la implementación, logros, metas. }\end{array}$ \\
\hline \multirow{2}{*}{ Análisis } & $\begin{array}{l}\text { Hace que los métodos y los datos que se utilizan en la herramienta estén al alcance } \\
\text { de todos. Favorece el análisis inmediato para la toma de decisiones. }\end{array}$ \\
\hline & $\begin{array}{l}\text { Facilita el trabajo de desarrollar una única "unidad de medida" para determinar } \\
\text { tendencias y evolución. }\end{array}$ \\
\hline \multirow{3}{*}{ Integración } & $\begin{array}{l}\text { Permite construir vínculos de relación compartida a través del reconocimiento por } \\
\text { participación. }\end{array}$ \\
\hline & $\begin{array}{l}\text { Facilita la Identificación de los Stakeholders objetivo de los cuales se requiere } \\
\text { feedback. }\end{array}$ \\
\hline & $\begin{array}{l}\text { Incrementa la participación, lo que permite compartir intereses, juicios, suposic } \\
\text { e interpretaciones frente a lasincertidumbres que reflejen los datos. }\end{array}$ \\
\hline
\end{tabular}

Tabla 4. Ventajas del cuadro de mando frente a los contextos relevantes de gestión 
La implementación del cuadro de mando como herramienta de coordinación horizontal proporciona a los administradores una mirada global de la organización desde diferentes perspectivas; su uso como soporte a la gestión es de alto valor, ya que permite controlar de forma continua si una empresa va a alcanzar los resultados definidos en el plan estratégico; también ayuda a la organización a expresar los objetivos e iniciativas necesarias para cumplir con la estrategia; es una herramienta que permite guiar tanto el desempeño actual como apuntar el desempeño futuro (Kaplan \& Norton, 1996, pp. 8).

La integración de todos los indicadores de desempeño facilita la movilización de esfuerzos a través de la canalización de intereses, relaciones, competencias y conocimientos específicos dentro de los MGC hacia el logro de metas estratégicas de largo plazo. En la Tabla 4 se expone las ventajas que se conseguirían con esta propuesta.

Se trata de una herramienta de control de gestión (Control), cuya función primordial es la unificación de los MGC (Integración), visualización del estado (Análisis) y notificación organizada (Comunicación) del nivel de avance de la empresa dentro de su estrategia de sostenibilidad (Desempeño). La comunicación es un aspecto esencial en todo proceso de unificación de sistemas y en este contexto es crítica; se habla de diferentes mecanismos de gestión que ejecutan diversidad de indicadores para evaluar sus planes y la comunicación de éstos a nivel externo e interno es esencial para ajustar la estrategia; en un trabajo previo los autores establecen como una buena gestión de la comunicación puede determinar el éxito o fracaso de la estrategia corporativa (Hurtado, Ferras, Arimany \& Meijide, 2015).

\section{Conclusiones}

La gestión de la estrategia de sostenibilidad es generalmente tratada como un proceso de emparejamiento del control del desempeño corporativo frente a criterios estándar de las agencias de calificación; pero debido a la heterogeneidad manifiesta tanto de las organizaciones como de sus condiciones de entorno hace más compleja la valoración de la organización y origina una falsa sensación de seguridad en inversores, entidades crediticias y en las partes interesadas; por tanto era necesario establecer elementos de consenso que dieran sentido a los actuales MGC y sirvieran para analizar su avance dentro de un mapa referencial.

Como objetivo de este estudio se planteó realizar una nueva asociación de ideas en torno a los MGC; para esto era necesaria la identificación de aspectos de gestión relevantes para la medición de logros, la descripción de la evolución y la construcción de estrategias corporativas en el ámbito de la sostenibilidad. Por consiguiente, se proyectó una investigación cualitativa a 
través de una exploración bibliográfica que tuviera como base el criterio de los autores de 90 artículos realizados entre los años 1989 y 2014; como factor de consenso se efectuó una clasificación de 3 subgrupos correspondientes a los conceptos más reconocidos y utilizados en la literatura de gestión de sostenibilidad en las organizaciones.

A partir de esta clasificación se logró identificar un total de 29 aspectos de marcado interés que hacían parte de los tres subgrupos; estos a su vez fueron agrupados en 6 contextos de acuerdo a su orientación, de esta manera se visualiza gráficamente el consenso de los tres grupos en relación a cada uno de los aspectos identificados; como resultado se pudo determinar que en el periodo de tiempo analizado existe una reiterada investigación orientada a contextos de Control, Integración, Análisis y Conceptos.

El contexto de "Control" está relacionado con los elementos necesarios para que exista una adecuada comprobación, inspección, fiscalización o intervención en los procesos de gestión de la sostenibilidad; el de "Integración" corresponde a la falta de elementos de cohesión dentro de la estructura operativa de la empresa. Con base en los contextos identificados, se propone como alternativa la implementación en la organización de un cuadro de mando como herramienta de coordinación horizontal; esta herramienta cumple en gran medida con el reto de asociar los indicadores de desempeño dentro de un único marco de observación y al mismo tiempo proporciona a los administradores una mirada global de la organización desde diferentes perspectivas, permitiéndoles ajustar la estrategia de sostenibilidad (Ravetz, 2000; Bonacchi \& Rinaldi, 2007; León-Soriano et al., 2010; Scipioni et al., 2009; Mori \& Christodoulou, 2012; Fegraus et al., 2012; Edgeman \& Williams, 2014).

La "Comunicación" cobra especial interés para llevar a cabo el "Control", "Integración" y "Análisis" de la gestión de la sostenibilidad en las prácticas empresariales; esto se debe a que mediante un modelo bidireccional se logra incrementar la participación, dialogo e interacción tanto del personal interno de la empresa, como de los stakeholders; de esta manera se posibilita el compartir y ajustar intereses, juicios, suposiciones e interpretaciones frente a las incertidumbres que puedan reflejar los datos (Sarvaiya \& Wu, 2014; Galbreath, 2014).

El estudio pone de relieve la importancia de basarse en la asociación de datos recogidos del análisis conjunto de los diferentes MGC que están directamente involucrados en la estrategia de sostenibilidad, así como los retos que implica el uso de dichos datos para ampliar los beneficios que su análisis aporta a la relación de sustentabilidad local-global.

Es importante señalar que aun con el mejor sistema de indicadores de un cuadro de control, esta herramienta necesita ser complementada con otro tipo de medidas (cualitativas/cuantitativas) e información adicional para asegurar un mayor impacto en las 
decisiones y los intereses de sustentabilidad de la organización a largo plazo; pero este análisis a profundidad va más allá del alcance de este estudio.

\section{Referencias}

ADAMCZYK, S.; BULLINGER, A.C.; MÖSLEIN, K.M. (2012). Innovation Contests: A Review, Classification and Outlook. Creativity and Innovation Management, 21(4): 335-360. http://dx.doi.org/10.1111/caim.12003

AGUINIS, H.; GLAVAS, A. (2012). What we know and don't know about corporate social responsibility: A review and research agenda. Journal of Management, 38: 932-968. http://dx.doi.org/10.1177/0149206311436079

ALONSO, P.A.; AZOFRA, V. (2008). El enfoque multistakeholder de la responsabilidad social corporativa: De la ambigüedad conceptual a la coacción y al intervencionismo. Revue Sciences de Gestion, Management Science, 66: 69-90.

ASIF, M.; SEARCY, C.; ZUTSHI, A.; AHMAD, N. (2011). An integrated management systems approach to corporate sustainability. European Business Review, 23(4): 353-367. http://dx.doi.org/10.1108/09555341111145744

ASIF, M.; SEARCY, C.; ZUTSHI, A.; FISSCHER, O. (2013). An integrated management systems approach to corporate social responsibility. Journal of Cleaner Production, 56: 7-17. http://dx.doi.org/10.1016/j.jclepro.2011.10.034

ATLEE, J.; KIRCHAIN, R. (2006). Operational sustainability metrics assessing metric effectiveness in the context of electronics-recycling systems. Environmental Science \& Technology, 40(14): 4506-4513. http://dx.doi.org/10.1021/es050935।

BANERJEE, S. (2008). Corporate social responsibility: The good, the bad and the ugly. Critical Sociology, 34: 51-79. http://dx.doi.org/10.1177/0896920507084623

BISBE, J. (2006). El uso de los sistemas de control de gestión por parte de la alta dirección, ¿bloquea o estimula la innovación?. Revista de Contabilidad y Dirección, 3: 177-194.

BOLIS, I.; MORIOKA, S.N.; SZNELWAR, L.I. (2014). When sustainable development risks losing its meaning. Delimiting the concept with a comprehensive literature review and a conceptual model. Journal of Cleaner Production, 83: 7-20. http://dx.doi.org/10.1016/j.jclepro.2014.06.041

BONACCHI, M.; RINALDI, L. (2007). DartBoards and Clovers as new tools in sustainability planning and control. Business Strategy and the Environment, 16: 461-473. http://dx.doi.org/10.1002/bse.596 
BOS-BROUWERS, H.E.J. (2010). Corporate sustainability and innovation in SMEs: Evidence of themes and activities in practice. Business Strategy and the Environment, 19(7): 417-435.

BRAMMER, S.J.; JACKSON, G.; MATTEN, D. (2012). Corporate Social Responsibility and Institutional Theory: New Perspectives on Private Governance. Socio Economic Review, 10(1): 3-28. http://dx.doi.org/10.1093/ser/mwr030

BRIASSOULIS, H. (2001). Sustainable Development and its Indicators: Through a (Planner's) Glass Darkly. Journal of Environmental Planning and Management, 44: 409-427. http://dx.doi.org/10.1080/09640560120046142

BROOK, J.W.; PAGANELLI, F. (2014). Integrating sustainability into innovation project portfolio management: A strategic perspective. Journal of Engineering and Technology Management, 34: 46-62. http://dx.doi.org/10.1016/j.jengtecman.2013.11.004

CHOO, C.W. (1996). The knowing organization: How organizations use information to construct meaning, create knowledge and make decisions. International Journal of Information Management, 16(5): 329-340. http://dx.doi.org/10.1016/0268-4012(96)00020-5

COYNE, K. (1986). Sustainable Competitive Advantage: What It Is, What It Isn't. Business Horizons, 29: 54-61. http://dx.doi.org/10.1016/0007-6813(86)90087-X

CRAMER, J. (2005). Applying international standards and guidelines on corporate social responsibility: An action plan. Environmental Quality Management, 14(3): 71-9. http://dx.doi.org/10.1002/tqem.20048

CRESWELL, J.W. (2007). Qualitative Inquiry \& Research Design - Choosing Among Five Approaches (Second edition, pp. 35-41). Thousand Oaks. CA: Sage.

DANCHEV, A. (2006). Social capital and sustainable behaviour of the firm. Industrial Management \& Data Systems, 106(7): 953-965. http://dx.doi.org/10.1108/02635570610688878

DYLLICK, T.; HOCKERTS, K. (2002). Beyond the business case for corporate sustainability. Business Strategy and the Environment, 11(2): 130-141. http://dx.doi.org/10.1002/bse.323

EDGEMAN, R.; WILLIAMS, J.A. (2014). Enterprise self-assessment analytics for sustainability, resilience and robustness. TQM Journal, 26(4): 368-381. http://dx.doi.org/10.1108/TQM-01-20140012

EPSTEIN, M.J.; ROY, M.J. (2001). Sustainability in action: Identifying and measuring the key performance drivers. Long Range Planning, 34(5): 585-604. http://dx.doi.org/10.1016/S00246301(01)00084-X

ESCRIG, E.; MUÑOZ, M.J.; FERNÁNDEZ, M.A. (2010). Socially responsible investing: Sustainability indices, ESG rating and information provider agencies. International Journal of Sustainable Economy, 2(4): 442-461. http://dx.doi.org/10.1504/IJSE.2010.035490 
FABER, N.; JORNA, R.J.; VAN ENGELEN, J. (2005). The Sustainability of "Sustainability". Journal of Environmental Assessment Policy and Management, 7(1): 1-33. http://dx.doi.org/10.1142/S1464333205001955

FEGRAUS, E.H.; ZASLAVSKY, I.; WHITENACK, T.; DEMPEWOLF, J.; AHUMADA, J.A.; LIN, K. ET AL. (2012). Interdisciplinary decision support dashboard: A new framework for a tanzanian agricultural and ecosystem service monitoring system pilot. IEEE Journal of Selected Topics in Applied Earth Observations and Remote Sensing, 5(6): 1700-1708.

FIGGE, F.; HAHN, T.; SCHALTEGgER, S.; WAGNER, M. (2002). The Sustainability Balanced Scorecard - linking sustainability management to business strategy. Business Strategy and the Environment, 11(5): 269-284. http://dx.doi.org/10.1002/bse.339

FRANDSEN, S.; MORSING, M.; VALLENTIN, S. (2013). Adopting Sustainability in the Organization: Managing Processes of Productive Loose Coupling Towards Internal Legitimacy. Journal of Management Development, 32(3): 236-246. http://dx.doi.org/10.1108/02621711311318265

GALBREATH, J. (2014). Building corporate social responsibility into strategy. European Business Review, 21(2): 109-127. http://dx.doi.org/10.1108/09555340910940123

GAZIULUSOY, İ.; BOYLE, C.; MCDOWALL, R. (2013). System innovation for sustainability: A systemic double-flow scenario method for companies. Journal of Cleaner Production, 45: 104-116. http://dx.doi.org/10.1016/j.jclepro.2012.05.013

GLOBAL REPORTING INITIATIVE.REPORTING 2025: AN INTERNATIONAL DIALOGUE. Disponible online en: https://www.globalreporting.org/information/Pages/Reporting-2025.aspx. (Fecha de la última consulta: 12 de marzo de 2015).

GOBBLE, M.M. (2014). Business Model Innovation. Research Technology Management, 57(6): 58.

GUO, Y.; YANG, D.C. (2014). Sustainability Accounting Reporting: A Survey on 30 U.S. DowJones Companies. International Journal of Accounting and Taxation, 2(3): 01-15

HAHN, T.; FIGGE, F. (2011). Beyond the Bounded Instrumentality in Current Corporate Sustainability Research: Toward an Inclusive Notion of Profitability. Journal of Business Ethics, 104(3): 325-345. http://dx.doi.org/10.1007/s10551-011-0911-0

HANSEN, E.G.; GROSSE-DUNKER, F.; REICHWALD, R. (2009). Sustainability innovation cube: A framework to evaluate sustainability-oriented innovations. International Journal of Innovation Management, 13(4): 683-713. http://dx.doi.org/10.1142/S1363919609002479 
HAUGH, H.M.; TALWAR, A. (2010). How do corporations embed sustainability across the organization. Academy of Management Learning and Education, 9(3): 384-396. http://dx.doi.org/10.5465/AMLE.2010.53791822

HURTADO, C.H.; FERRAS, X.; ARIMANY, N.; MEIJIDE, D. (2015, June). Communications and corporate social responsibility: A canvas to build its strategy. In Information Systems and Technologies (CISTI), 2015 10th Iberian Conference on (pp. 1-8). http://dx.doi.org/10.1109/CISTI.2015.7170551

HJORTHA, P.; BAGHERIA, A. (2006). Navigating towards sustainable development: A system dynamics approach. Futures, 38: 74-92. http://dx.doi.org/10.1016/j.futures.2005.04.005

ISAKSSON, R. (2006). Total quality management for sustainable development: Process based system models. Business Process Management Journal, 12(5): 632-645. http://dx.doi.org/10.1108/14637150610691046

KAPLAN, R.S.; DAVID, P.N. (1996). The Balanced Scorecard - Translating Strategy Into Action. Boston, MA: Harvard Business School Press.

KATRIN, S.; KEHRBACH, F. (2014). A three-dimensional framework to explore corporate sustainability activities in the mining industry: Current status and challenges ahead. Resources Policy, 46(1): 101-115. http://dx.doi.org/10.1016/j.resourpol.2014.10.009

KOLK, A. (2004). A decade of sustainability reporting: developments and significance. International Journal of Environment and Sustainable Development, 3(1), 51-64. http://dx.doi.org/10.1504/IJESD.2004.004688

LEE, K.-H. (2011). Motivations, barriers, and incentives for adopting environmental management (cost) accounting and related guidelines: A study of the Republic of Korea. Corporate Social Responsibility and Environmental Management, 18(1), 39-49. http://dx.doi.org/10.1002/csr.239

LEE, K.-H.; FARZIPOORSAEN, R. (2012). Measuring corporate sustainability management: A data envelopment analysis approach. International Journal of Production Economics, 140(1): 219-226. http://dx.doi.org/10.1016/j.ijpe.2011.08.024

LEÓN-SORIANO, R; MUÑOZ-TORRES, M.J.; CHALMETA-ROSALEN, R. (2010). Methodology for sustainability strategic planning and management. Industrial Management \& Data Systems, 110(2): 249-268. http://dx.doi.org/10.1108/02635571011020331

LOORBACH, D. (2010). Transition management for sustainable development: A prescriptive, complexity-based governance framework. Governance, 23(1): 161-183. http://dx.doi.org/10.1111/j.1468-0491.2009.01471.x 
MARREWIJK, M. (2003). Concepts and Definitions of CSR and Corporate Sustainability: Between Agency and Communion. Journal of Business Ethics, 44(2-3): 95-105. http://dx.doi.org/10.1023/A:1023331212247

MOHAMAD, F.; ABDULLLAH, N.H.; MOHAMMAD, M.; KAMARUDDIN, N.K. (2014). Management systems integration for organizational sustainability: Quality, environmental, occupational health and safety, and energy. Applied Mechanics and Materials, 465-466: 1155-1159. http://dx.doi.org/10.4028/www.scientific.net/AMM.465-466.1155

MONEVA, J.M.; ARCHEL, P.; CORREA, C. (2006). GRI and the camouflaging of corporate unsustainability. Accounting Forum, 30(2): 121-137. http://dx.doi.org/10.1016/j.accfor.2006.02.001

MONTIEL, I. (2008). Corporate social responsibility and corporate sustainability: Separate pasts, common futures. Organization and Environment, 21(3): 245-269. http://dx.doi.org/10.1177/1086026608321329

MONTIEL, I.; DELGADO-CEBALLOS, J. (2014). Defining and Measuring Corporate Sustainability: Are We There Yet?. Organization and Environment, 27(2): 113-139. http://dx.doi.org/10.1177/1086026614526413

MORI, K.; CHRISTODOULOU, A. (2012). Review of sustainability indices and indicators: Towards a new City Sustainability Index (CSI). Environmental Impact Assessment Review, 32(1): 94-106. http://dx.doi.org/10.1016/j.eiar.2011.06.001

OCAMPO, L.A.; CLARK, E. (2014). Developing a framework for sustainable manufacturing strategies selection. DLSU Business and Economics Review, 23(2): 115-131.

PORTER, M.E.; KRAMER, M.R. (2006). Strategy \& society: The link between competitive advantage and corporate social responsibility. Harvard Business Review, 84(12): 78-92.

RAMOS, T.B.; CAEIRO, S.D. (2010). Meta-performance evaluation of sustainability indicators. Ecological Indicators, 10(2), 157-166. http://dx.doi.org/10.1016/j.ecolind.2009.04.008

RAVETZ, J. (2000). Integrated assessment for sustainability appraisal in cities and regions. Environmental Impact Assessment Review, 20(1): 31-64. http://dx.doi.org/10.1016/S01959255(99)00037-2

RICCABONI, A; LEONE, E.L. (2011). Implementing strategies through management control systems: The case of sustainability. International Journal of Productivity and Performance Management, 59(2): 130-144. http://dx.doi.org/10.1108/17410401011014221

ROSS, A. (2009). Modern Interpretations of Sustainable Development. Journal of Law and Society, 36(1): 32-54. http://dx.doi.org/10.1111/j.1467-6478.2009.00455.x 
SARVAIYA, H.; WU, M. (2014). An integrated approach for corporate social responsibility and corporate sustainability. Asian Social Science, 10(17): 57-70. http://dx.doi.org/10.5539/ass.v10n17p57

SCHAEFER, A. (2007). Contrasting Institutional and Performance Accounts of Environmental Management Systems: Three Case Studies in the UK Water \& Sewerage Industry. Journal of Management Studies, 44: 507-535. http://dx.doi.org/10.1111/j.1467-6486.2006.00677.x

SCHALTEGGER, S.; BURRITT, R.L. (2010). Sustainability accounting for companies: Catchphrase or decision support for business leaders?. Journal of World Business, 45(4): 375-384. http://dx.doi.org/10.1002/bse.1772

SCHALTEGgER, S.; BECKMANN, M.; HANSEN, E.G. (2013). Transdisciplinarity in Corporate Sustainability: Mapping the Field. Business Strategy and the Environment, 22(4): 219-229. http://dx.doi.org/10.1016/j.jwb.2009.08.002

SCHERER, A.G.; PALAZZO, G. (2011). The new political role of business in a globalized world: A review of a new perspective on CSR and its implications for the firm, governance, and democracy. Journal of management studies, 48(4), 899-931. http://dx.doi.org/10.1111/j.14676486.2010.00950.x

SCHRETTLE, S.; HINZ, A.; SCHERRER-RATHJE, M.; FRIEDLI, T. (2014). Turning sustainability into action: Explaining firms' sustainability efforts and their impact on firm performance. International Journal of Production Economics, 147 (PART A): 73-84.

SCHWARTZ, M.S.; CARROLL, A.B. (2008). Integrating and unifying competing and complementary frameworks: The search for a common core in the business and society field. Business and Society, 47(2): 148-186. http://dx.doi.org/10.1177/0007650306297942

SCIPIONI, A.; MAZZI, A.; MASON, M.; MANZARDO, A. (2009). The Dashboard of Sustainability to measure the local urban sustainable development: The case study of Padua Municipality. Ecological Indicators, 9(2): 364-380. http://dx.doi.org/10.1016/j.ecolind.2008.05.002

SHRIVASTAVA, P.; HART, S. (1995). Creating Sustainable Corporations. Business Strategy and the Environment, 4: 154-165. http://dx.doi.org/10.1002/bse.3280040307

SIEGEL, S. (2009). Green management matters only if it yields more green: AN economic/strategic perspective. Academy of Management Perspectives, 23(3): 5-16. http://dx.doi.org/10.5465/AMP.2009.43479260

SIEBENHÜNER, B; ARNOLD, M. (2007). Organizational learning to manage sustainable development. Business strategy and the environment, 16(5), 339-353. http://dx.doi.org/10.1002/bse.579 
SUSTAINABILITY (2013). The 2013 Ratings Survey. Polling the Experts. A GlobeScan/SustainAbility Survey. Rate of raters. 13 March 2013.

VIGNEAU, L.; HUMPHREYS, M.; MOON, J. (2014). How Do Firms Comply with International Sustainability Standards? Processes and Consequences of Adopting the Global Reporting Initiative. Journal of Business Ethics, 131(2): 469-486. http://dx.doi.org/10.1007/s10551-014-22785

VINAYAN, G.; JAYASHREE, S.; MARTHANDAN, G. (2012). Critical Success Factors of Sustainable Competitive Advantage: A Study in Malaysian Manufacturing Industries. International Journal of Business and Management, 7(22): 29. http://dx.doi.org/10.5539/ijbm.v7n22p29

WIEDMANN, T.O.; LENZEN, M.; BARRETT, J.R. (2009). Companies on the scale comparing and benchmarking the sustainability performance of businesses. Journal of Industrial Ecology, 13(3): 361-383. http://dx.doi.org/10.1111/j.1530-9290.2009.00125.x

Intangible Capital, 2016 (www.intangiblecapital.org) 